\title{
A REGULARIZING EFFECT OF NONLINEAR TRANSPORT EQUATIONS
}

\author{
$\mathrm{BY}$ \\ FELIX OTTO \\ Institut für Angewandte Mathematik der Universität Bonn, Germany
}

Abstract. We consider the semigroup on $L^{1}\left(\mathbb{R}^{n}\right)$ defined by the nonlinear transport equation for the scalar $s$,

$$
\partial_{t} s+\operatorname{div}(f(s) u)=0 \quad \text { in }(0, \infty) \times \mathbb{R}^{n}
$$

for given velocity field $u$. We show that this nonlinear semigroup is Hölder continuous for $t>0$ in the uniform operator topology, provided the graph of $f$ has no linear segments. This continuity property - which expresses a regularizing effect of the nonlinearity in the transport equation - is robust with respect to the spatial behaviour of the timeindependent velocity field $u$.

Introduction. Consider a scalar quantity $s$ - think of the density of some continuum-

$$
s(t, x)
$$

depending on time $t \in(0, \infty)$ and location $x \in \mathbb{R}^{n}$. Let us assume that $s$ evolves in time according to a flux of the following structure:

$$
f(s) u(t, x)
$$

with given flux function $f: \mathbb{R} \rightarrow \mathbb{R}$ and velocity field $u:(0, \infty) \times \mathbb{R}^{n} \rightarrow \mathbb{R}^{n}$. This yields a formal evolution equation for $s$

$$
\begin{aligned}
\partial_{t} s+\operatorname{div}(f(s) u) & =0 & & \text { in } Q:=(0, \infty) \times R^{n}, \\
s(t=0) & =s^{0} & & \text { on } \mathbb{R}^{n},
\end{aligned}
$$

where $s^{0}$ are some initial data.

If $f$ is nonlinear, two well-known effects occur:

- An initially smooth solution of (1) may develop a singularity in finite time, a so-called shock.

- A distributional solution of (1) may not be unique.

Received October 10, 1995.

1991 Mathematics Subject Classification. Primary 35F25, 35L65. 
So we have to be more precise about what we understand by the evolution operator defined by (1): It is the strong limit of the evolution operators defined by

$$
\begin{aligned}
& \partial_{t} s+\operatorname{div}(f(s) u)-D \Delta s=0 \quad \text { in } Q, \\
& s(0)=s^{0} \text { on } \mathbb{R}^{n},
\end{aligned}
$$

for $D \downarrow 0$, the appropriate space that all these operators act on being $L^{1}\left(\mathbb{R}^{n}\right)$. This "viscosity" approach leads to the concept of entropy solutions; its well-posedness has been proved by Kružhov [11].

Equations of type (1) arise as a component of porous media flow models, for instance,

- in saturated-unsaturated water flow through a porous medium, where

- $s$ is the saturation,

- $f$ is the mobility of the fluid,

- $u$ is the permeability matrix of the medium $\times(-$ pressure gradient + gravity vector),

- in two-phase fluid flow through porous media, where

- $s$ is the fractional amount of one of the fluids,

- $f$ is the corresponding "fractional flow function",

- $u$ is the total seepage velocity.

In this article, we will look for some regularizing effect of Eq. (1) that is robust with respect to the spatial regularity of $u$. For simplicity, we will from now on assume that $u$ does not depend on time. More precisely: we will investigate some continuity property of the semi-group defined by (1) which is essentially independent of the spacial behaviour of $u$. What kind of regularity effect may we expect? First of all, if $f$ is affine on some interval $I$, there is no regularizing effect at all: solving (1) for some initial data $s^{0}$ with range in $I$ just means translating $s^{0}$ along the streamlines of $u$. In any case, there is no regularizing effect transversal to the streamlines of $u$ : it is quite obvious that $\left.s(t)\right|_{\omega}$ depends only on $\left.s^{0}\right|_{\omega}$ for any streamline $\omega$ of $u$. There may be some regularizing effect in space along each streamline, but it of course depends on $u$. So the only regularizing effect that has a chance to be independent of the spatial behaviour of $u$ is one in time - and it will heavily depend on the graph of $f$ having no linear segments. This is essentially what we can prove.

Result. We actually need a more technical version of the condition that the graph of $f$ contains no linear segments.

Definition. A smooth function $f: \mathbb{R} \rightarrow \mathbb{R}$ is called strictly nonlinear if and only if 1. $f^{\prime \prime}$ vanishes only in finitely many points and of finite order;

2. there exist $M<\infty$ and $a_{ \pm}, b_{ \pm} \in \mathbb{R}$ such that

$$
f^{\prime}(z)=\left\{\begin{array}{ll}
a_{+} z^{p_{+}} & \text {for } z \geq M \\
a_{-}(-z)^{p_{-}} & \text {for } z \leq-M
\end{array}\right\}
$$


TheOREM. Let $f: \mathbb{R} \rightarrow \mathbb{R}$ be strictly nonlinear with $f(0)=f^{\prime}(0)=0$ and let $u: \mathbb{R}^{n} \rightarrow$ $\mathbb{R}^{n}$ be smooth with compact support. Let $s$ be the entropy solution of

$$
\begin{aligned}
\partial_{t} s+\operatorname{div}(f(s) u) & =0 & & \text { in } Q, \\
s(0) & =s^{0} & & \text { on } \mathbb{R}^{n} .
\end{aligned}
$$

Then for all $0<\lambda \leq 1$ and $t>0$

$$
\|s(t)-s(\lambda t)\|_{L^{1}\left(R^{n}\right)} \leq C(1-\lambda)^{\alpha}\left(\left\|s^{0}\right\|_{L^{1}\left(R^{n}\right)}+t\|\operatorname{div} u\|_{L^{1}\left(R^{n}\right)}\right),
$$

where $0<\alpha$ and $C<\infty$ depend only on $f$.

REMARKS.

a) If $\operatorname{div} u=0$ or if $f$ has some special structure, there are a priori estimates for the $L^{\infty}(Q)$-norm of $s$ in terms of $\left\|s^{0}\right\|_{L^{\infty}\left(R^{n}\right)}$. In this case we may skip part 2) of the Definition, with the cost of allowing $C$ to depend on $\left\|s^{0}\right\|_{L^{\infty}\left(R^{n}\right)}$.

b) The condition $f(0)=f^{\prime}(0)=0$ is not essential. All we need to obtain a similar result is the existence of a $z_{0}$ such that $f^{\prime}\left(z_{0}\right)=0$.

c) Observe that the result only depends on $u$ via the $L^{1}$-norm of its divergence. So by continuity, the result can be extended to a large class of velocity fields since (1) remains well-posed and still continuously depends on $u$.

d) Observe that the metric on $(0, \infty)$ by which we measure the Hölder continuity of the map $s:(0, \infty) \rightarrow L^{1}\left(\mathbb{R}^{n}\right)$ is given by

$$
\left|1-\frac{t_{1}}{t_{2}}\right|
$$

e) Notice that the estimate of the modulus of continuity of $s:(0, \infty) \rightarrow L^{1}\left(\mathbb{R}^{n}\right)$ (with respect to the metric (2)) only depends on $f,\left\|s^{0}\right\|_{L^{1}\left(R^{n}\right)}$, and $\|\operatorname{div} u\|_{L^{1}\left(R^{n}\right)}$. As a consequence,

- high oscillation of the initial data do not affect the estimate,

- superposition of $u$ with a highly oscillating incompressible velocity field does not alter the estimate.

f) An explicit investigation of one-dimensional examples will convince the reader that a subtle interaction of shocks and rarefaction waves is the mechanism that eventually dampens the amplitude of initial oscillations - the rate of decay depending on the order of the critical points of $f^{\prime}$. But as opposed to other studies of the qualitative behaviour of solutions of conservation laws $[9,10,4,8,3,18]$, our proof makes no use of this geometric intuition.

g) The proof (see Lemma 3) yields an explicit formula for the Hölder exponent $\alpha$ :

$$
\alpha:=\frac{1}{m+1},
$$

where $m$ is related to the order of those critical points of $f^{\prime}$ that are not zeros:

$$
m:=\max \left[\{ 0 \} \cup \left\{k \geq 2 \mid \text { there exists a } z_{0} \in \mathbb{R}\right.\right. \text { such that }
$$

$$
\left.\left.f^{\prime}\left(z_{0}\right) \neq 0, f^{\prime \prime}\left(z_{0}\right)=\cdots=f^{(k)}\left(z_{0}\right)=0, f^{(k+1)}\left(z_{0}\right) \neq 0\right\}\right] .
$$

h) The assumption that $u$ does not depend on $t$ is essential: an explicit construction in one space dimension shows that a velocity field that is constant in space but 
rapidly changes sign in time (with mean zero) prevents shocks and rarefaction waves from interacting.

i) The method introduced in the sequel to prove this result may also be applied to prove a regularizing effect in space in the case of constant $u$ and one space dimension.

Discussion. Let us now discuss how our result relates to prior works. To our knowledge, it is not contained in prior ones since those either assume that

- $u$ is constant

or

- $f$ has "special structure".

If $u$ is constant, the equation of course reduces effectively to a homogeneous scalar conservation law in one space dimension. By $f$ having "special structure", we understand that

- $f$ is strictly convex,

- $f$ is homogeneous, i.e.,

$$
f^{\prime}(z)=\left\{\begin{array}{ll}
a_{+} z^{p} & \text { for } z \geq 0 \\
a_{-}(-z)^{p} & \text { for } z \leq 0
\end{array}\right\}
$$

for some $a_{ \pm} \in \mathbb{R}$ and $p \neq 0$,

or, loosely speaking,

- a combination of both.

In any case, special structure admits a Hölder exponent $\alpha=1$. Not included are $f$ 's that allow for a $z_{0} \in \mathbb{R}$ such that

$$
f^{\prime}\left(z_{0}\right) \neq 0, \quad f^{\prime \prime}\left(z_{0}\right)=0, \quad \text { and } \quad f^{\prime \prime \prime}\left(z_{0}\right) \neq 0
$$

- the most common fractional flow function in immiscible two-phase flow in porous media just exhibits this behaviour.

Probably the first result for constant $u$ and strictly convex $f$ is due to Oleinik [15]: her E-condition that she used to characterize the entropy solutions of scalar conservation laws in one space dimension just expresses the regularizing effect in a straightforward way. A first qualitative version of the regularizing effect for constant $u$ and general (i.e., strictly nonlinear) $f$ is given by Dafermos' result [6] on the asymptotic behaviour of solutions of a scalar conservation law in one space dimension with periodic initial data. He works in the setting of dynamical systems. Another purely qualitative version of the regularizing effect for constant $u$ and general $f$ is Tartar's compactness result for scalar conservation laws in one space dimension [17]. His tools are compensated compactness and Young measures. Lions, Perthame, and Tadmor [13]-using their kinetic formulation of scalar conservation laws, which allows for an application of the velocity averaging principle, were able to prove a regularizing effect of nonlinearity in scalar conservation laws in several space dimensions. In the case of constant $u$ and general $f$, their result can be applied to our setting and yields

$$
\|s\|_{L_{\tau}^{p}\left((\varepsilon, 1 / \varepsilon), L^{p}\left(B_{1 / \varepsilon}\right)\right)} \leq C(f, \varepsilon)\left\|s^{0}\right\|_{L^{1}\left(R^{n}\right)}
$$

- some fractional time derivative is locally estimated in some $L^{p}$-norm. Since the velocity averaging principle is based on Fourier transforms in space and time, their result is 
nonlocal in time (and definitely not optimal with respect to the order of Besov space). On the other hand, by fully exploiting the $L^{1}$-contraction principle for scalar conservation laws, Bénilan and Crandall ([1], see also [5]) established exactly our result in the case of general $u$ (at least implicitly) but special structure $f$. In a sense, our result is also a weak form of asymptotic analysis and therefore related to the studies of the asymptotic behaviour of solutions of conservation laws in one space dimension like [12, Theorem 6], [9, Theorem 5.2], [6], [10, Theorem 3], [7, Theorem 7.1], [4, Theorem 2.2], [14, Theorems 3.1, $3.2,3.3],[8],[3$, Theorems 4.4, 5.15], [18, Theorem 2.1], who apart from the qualitative approach in [6] assume that $f$ has some kind of special structure.

Is it possible to reduce the case of general $u$ or $f$ to that of constant $u$, resp. special structure $f$ ? If $u$ is incompressible (meaning $\operatorname{div} u=0$ ), this is achievable, albeit clumsy. But this reduction seems to be not feasible when div $u$ does not vanish. This shows that our result is more one of advection than of homogeneous scalar conservation laws. On the other hand, if $f^{\prime}$ has a single critical point, our setting can be transformed into the special structure case, generating a somewhat poorer result. If $f$ has more than one inflection point, this reduction is no longer viable.

We employ ideas from both sides: We make use of Lions, Perthame, and Tadmor's kinetic formulation (Lemma 1) and mimic Bénilon and Crandall's application of the $L^{1}$-contraction principle (Lemma 2).

Proof. Fix $\lambda \in(0,1)$. We want to compare $s$ to its rescaled version in time, $s(\lambda t, x)$. If $f$ is homogeneous, i.e., $f(z)=z^{p}$, we are lucky since

$$
\tilde{s}(t, x):=\lambda^{\frac{1}{p-1}} s(\lambda t, x)
$$

is again an entropy solution of (1). In the general case, we will show that a nonlinear transformation

$$
\tilde{s}(t, x):=\Phi_{\lambda}(s(\lambda t, x))
$$

does a good job. To give an idea of this, let us just for the moment assume that

A1) there exists no $z_{0} \in \mathbb{R}$ such that $f^{\prime}\left(z_{0}\right) \neq 0$ and $f^{\prime \prime}\left(z_{0}\right)=0$;

A2) $\operatorname{div} u=0$;

A3) $s$ is a smooth solution.

Because of $A 1$,

$$
f^{\prime}\left(\Phi_{\lambda}(z)\right)=\lambda f^{\prime}(z)
$$

defines a homomorphism $\Phi_{\lambda}: \mathbb{R} \rightarrow \mathbb{R}$ (see also Lemma 3). It is constructed such that (3) transforms $s$, which by assumption A3 is a smooth solution, into a smooth solution $\tilde{s}$ of Eq. (1) - here we also use A2 (and the time-independence of $u$ ). So by the $L^{1}$-contraction principle ([11], see also Lemma 2), we obtain for all $t \geq 0$

$$
\|s(t)-\tilde{s}(t)\|_{L^{1}\left(R^{n}\right)} \leq\|s(0)-\tilde{s}(0)\|_{L^{1}\left(R^{n}\right)},
$$

yielding

$$
\|s(t)-s(\lambda t)\|_{L^{1}\left(R^{n}\right)} \leq \sup _{z \in R}\left|\frac{1}{z}\left(\Phi_{\lambda}(z)-z\right)\right|\left(\|s(\lambda t)\|_{L^{1}\left(R^{n}\right)}+\|s(0)\|_{L^{1}\left(R^{n}\right)}\right) .
$$


This entails the claim, since by calculus methods (see also Lemma 3 )

$$
\sup _{z \in R}\left|\frac{1}{z}\left(\Phi_{\lambda}(z)-z\right)\right| \leq C(1-\lambda)
$$

and-once again by the $L^{1}$-contraction principle-

$$
\|s(\lambda t)\|_{L^{1}\left(R^{n}\right)} \leq\|s(0)\|_{L^{1}\left(R^{n}\right)} .
$$

Our proof now consists in getting rid of the additional assumptions A1, A2, and A3. Because of the $L^{1}$-contraction principle, we may without loss of generality assume that $s^{0} \in C_{0}^{\infty}\left(\mathbb{R}^{n}\right)$. $\|\cdot\|$ will denote the $L^{1}\left(\mathbb{R}^{n}\right)$-norm throughout the sequel. Let $\Psi: \mathbb{R} \rightarrow \mathbb{R}$ be such that $\Psi^{\prime \prime}$ has compact support and $\Psi(0)=0$. Let $\tilde{f}$ be defined by

$$
\tilde{f}^{\prime}(\Psi(z))=f^{\prime}(z) \text { and } \tilde{f}(0)=0 .
$$

If $s$ is a smooth solutions of $(1)$, then $\tilde{s}=\Psi(s)$ is a smooth solution of

$$
\partial_{t} \tilde{s}+\operatorname{div}(\tilde{f}(\tilde{s}) u)=0 .
$$

But if $s$ is only an entropy solution of (1), $\tilde{s}$ generally is not an entropy solution of (4) (that is why A3 was so helpful). So we have to investigate the effect of the nonlinear transformation $\Psi$ on the entropy solutions of Eq. (1). In order to avoid technicalities, we investigate this effect for solutions of the viscosity approximation of (1); the following lemma is inspired by the kinetic formulation of Lions, Perthame, and Tadmor [13].

LEMMA 1. Let $s$ be a (classical) solution of

$$
\partial_{t} s+\operatorname{div}(f(s) u)-D \Delta s=0 .
$$

Then $\tilde{s}:=\Psi(s)$ is a solution of

$$
\partial_{t} \tilde{s}+\operatorname{div}(\tilde{f}(\tilde{s}) u)-D \Delta \tilde{s}=e,
$$

where the right-hand side is estimated as follows:

$$
\|e\|_{L^{1}\left(Q_{T}\right)} \leq \int_{R}\left|\Psi^{\prime \prime}(z)\right| d z\|s(0)\|+2 \int_{R}\left|\Psi^{\prime \prime}(z) f(z)\right| d z T\|\operatorname{div} u\| .
$$

Proof. The result relies on the fact that $e$ is essentially non-positive if $\Psi$ is convex. Obviously

$$
-e=D \Psi^{\prime \prime}(s)|\nabla s|^{2}+\left(\Psi^{\prime}(s) f(s)-\tilde{f}(\Psi(s))\right) \operatorname{div} u .
$$

Because of

$$
\begin{aligned}
\frac{d}{d z}\left[\Psi^{\prime}(z) f(z)-\tilde{f}(\Psi(z))\right] & =\psi^{\prime \prime}(z) f(z), \\
{\left[\Psi^{\prime}(0) f(0)-\tilde{f}(\Psi(0))\right] } & =0
\end{aligned}
$$

we infer

$$
\left\|\left(\Psi^{\prime}(s) f(s)-\tilde{f}(\Psi(s))\right) \operatorname{div} u\right\|_{L^{1}\left(Q_{T}\right)} \leq \int_{R}\left|\Psi^{\prime \prime}(z) f(z)\right| d z T\|\operatorname{div} u\| .
$$

We now claim that for continuous $h: \mathbb{R} \rightarrow \mathbb{R}$ with compact support

$$
\left\|D h(s)|\nabla s|^{2}\right\|_{L^{1}\left(Q_{T}\right)} \leq \int_{R}|h(z)| d z\|s(0)\|+\int_{R}|h(z) f(z)| d z T\|\operatorname{div} u\| .
$$


Without loss of generality we may assume $h \geq 0$. Let $\Psi_{h}$ and $\tilde{f}_{h}$ be defined by

$$
\begin{aligned}
& \Psi_{h}^{\prime \prime}=h \quad \text { and } \quad \Psi_{h}^{\prime}(0)=\Psi_{h}(0)=0, \\
& \tilde{f}^{\prime}\left(\Psi_{h}(z)\right)=f^{\prime}(z) \text { and } \tilde{f}_{h}(0)=0 .
\end{aligned}
$$

By the same calculation that led to (5), we obtain for $\tilde{s}_{h}:=\Psi_{h}(s)$,

$$
D h(s)|\nabla s|^{2}=-\left\{\partial_{t} \tilde{s}_{h}+\operatorname{div}\left(\tilde{f}_{h}\left(\tilde{s}_{h}\right) u\right)-D \Delta \tilde{s}_{h}\right\}-\left(\Psi_{h}^{\prime}(s) f(s)-\tilde{f}_{h}\left(\Psi_{h}(s)\right)\right) \operatorname{div} u .
$$

Hence

$\left\|D h(s)|\nabla s|^{2}\right\|_{L^{1}\left(Q_{T}\right)}=\int_{R^{n}} \Psi_{h}(s(0))-\int_{R^{n}} \Psi_{h}(s(T))-\int_{Q_{T}}\left(\Psi_{h}^{\prime}(s) f(s)-\tilde{f}_{h}\left(\Psi_{h}^{\prime}(s)\right)\right) \operatorname{div} u$.

Because of

$$
0 \leq \Psi_{h}(z) \leq|z| \int_{R} h(\tilde{z}) d \tilde{z}
$$

and

$$
\left|\int_{Q_{T}}\left(\Psi_{h}^{\prime}(s) f(s)-\tilde{f}_{h}\left(\Psi_{h}^{\prime}(s)\right)\right) \operatorname{div} u\right| \leq \int_{R}|h(z) f(z)| d z T\|\operatorname{div} u\|
$$

-which is the same estimate as (6)-we obtain (7). We now apply (7) to $h=\Psi^{\prime \prime}$ and infer from (5), (6), and (7) the claim of the lemma.

If $\mathrm{A} 1$ is no longer valid, there might be no diffeomorphism $\Phi: \mathbb{R} \rightarrow \mathbb{R}$ such that

$$
f^{\prime}(\Phi(z))=\lambda f^{\prime}(z) \quad \text { for all } z \in \mathbb{R} .
$$

Even if there is one (in case $f^{\prime \prime}$ does not change sign), the total variation of the derivative will be infinite:

$$
\int_{R}\left|\Phi^{\prime \prime}(z)\right| d z=\operatorname{TV}\left(\Phi^{\prime}\right)=\infty
$$

and hence Lemma 1 is of no direct use.

Thus we have to relax condition (8). The idea is to construct a diffeomorphism $\Phi: \mathbb{R} \rightarrow \mathbb{R}$ such that (8) is satisfied except in small intervals around the points $z_{0} \in \mathbb{R}$ with

$$
f^{\prime}\left(z_{0}\right) \neq 0 \text { and } f^{\prime \prime}\left(z_{0}\right)=0 .
$$

The length of those intervals will tend to zero as $\lambda$ tends to one. Let us start by fixing a diffeomorphism $\Phi: \mathbb{R} \rightarrow \mathbb{R}$ with the properties

$$
\Phi^{\prime \prime} \text { has compact support and } \Phi(0)=0
$$

( $\Phi=\Phi_{\lambda}$ will be constructed in Lemma 3$)$. We measure the deviation from (8) by a function $g: \mathbb{R} \rightarrow \mathbb{R}$ that is defined by

$$
g^{\prime}(\Phi(z))=\frac{f^{\prime}(\Phi(z))}{\lambda f^{\prime}(z)} \quad \text { and } \quad g(0)=0 .
$$

In Lemma $3, \Phi$ will be constructed in such a way that $g$ is well defined and

$$
g^{\prime}(z) \in\left[\lambda, \frac{1}{\lambda}\right] \text { for all } z \in \mathbb{R} \text { and } g(z)=1 \text { for }|z| \gg 1 \text {. }
$$


This particular way of measuring the deviation from (8) is motivated by the following consideration: If $s$ is a smooth solution of (1) then by construction of $g, \tilde{s}(t, x)=\Phi(s(\lambda t, x))$ would be a smooth solution of

$$
\partial_{t}(g(\tilde{s}))+\operatorname{div}(f(\tilde{s}) u)=0 .
$$

So albeit $s$ and $\tilde{s}$ do not satisfy the same equation, the respective equations differ only in their $\partial_{t}$-part. This is crucial because only perturbations of the $\partial_{t}$-part can be balanced by the regularizing effect in time.

Let $s$ be an entropy solution of (1). We use the fact that $s$ is the limit in $C^{0}([0, T]$, $\left.L^{1}\left(\mathbb{R}^{n}\right)\right)$ of both sequences $\left(s_{D}\right)_{D \downarrow 0}$ and $\left(s_{D}^{*}\right)_{D \downarrow 0}$ defined by

$$
\begin{aligned}
\partial_{t} s_{D}+\operatorname{div}\left(f\left(s_{D}\right) u\right)-D \Delta s_{D} & =0, \\
s_{D}(0) & =s^{0},
\end{aligned}
$$

resp.

$$
\begin{aligned}
\partial_{t} s_{D}^{*}+\operatorname{div}\left(f\left(s_{D}^{*}\right) u\right)-\lambda D \Delta\left(g\left(s_{D}^{*}\right)\right) & =0, \\
s_{D}^{*}(0) & =s^{0}
\end{aligned}
$$

(recall that $g$ is strictly increasing). We consider

$$
\begin{aligned}
& \tilde{s}_{D}(t, x):=\Phi\left(s_{D}(\lambda t, x)\right), \\
& \bar{s}_{D}(t, x):=g\left(\tilde{s}_{D}(t, x)\right)=(g \circ \Phi)\left(s_{D}(\lambda t, x)\right) .
\end{aligned}
$$

Due to Lemma $1, \bar{s}_{D}$ is a solution of

$$
\left\{\frac{1}{\lambda} \partial_{t} \bar{s}_{D}+\operatorname{div}\left(\bar{f}\left(\bar{s}_{D}\right) u\right)-D \Delta \bar{s}_{D}\right\}(t, x)=\bar{e}(\lambda t, x)
$$

(here we have used time-independence of $u$ ) where the flux $\bar{f}$ is given by

$$
\bar{f}^{\prime}((g \circ \Phi)(z))=f^{\prime}(z) \text { and } \bar{f}(0)=0
$$

and the right-hand side $\bar{e}$ is estimated as follows:

$$
\|\bar{e}\|_{L^{1}\left(Q_{\lambda T}\right)} \leq \int_{R}\left|(g \circ \Phi)^{\prime \prime}(z)\right| d z\left\|s^{0}\right\|+2 \int_{R}\left|(g \circ \Phi)^{\prime \prime}(z) f(z)\right| d z \lambda T\|\operatorname{div} u\| .
$$

By construction, this means in terms of $\tilde{s}_{D}$

$$
\partial_{t}\left(g\left(\tilde{s}_{D}\right)\right)+\operatorname{div}\left(f\left(\tilde{s}_{D}\right) u\right)-\lambda D \Delta\left(g\left(\tilde{s}_{D}\right)\right)=e
$$

with the right-hand side bounded by

$$
\|e\|_{L^{1}\left(Q_{T}\right)} \leq \int_{R}\left|(g \circ \Phi)^{\prime \prime}(z)\right| d z\left\|s^{0}\right\|+2 \int_{R}\left|(g \circ \Phi)^{\prime \prime}(z) f(z)\right| d z \lambda T\|\operatorname{div} u\| .
$$

Unlike the case $g=\mathrm{id}$, even if $e \equiv 0$, we cannot prove that $\left\|s_{D}^{*}(t)-\tilde{s}_{D}(t)\right\|$ is nonincreasing in time. Nevertheless, we are able to show that the growth of some averaged quantity

$$
\int_{R}\left\|s_{D}^{*}(t+\tau)-\tilde{s}_{D}(t-\tau)\right\| \phi(\tau) d \tau
$$

is controlled by $e$ and the sup-norm of $g$ - id times the inverse of the typical length of the averaging variable $\tau$ :

$$
\sup _{z \in R}|g(z)-z| \int_{R}\left|\phi^{\prime}(\tau)\right| d \tau \text {. }
$$


We use a technique introduced by Kružkov [11] (see also [16]) to prove uniqueness for scalar conservation laws: the doubling variables.

LEMMA 2. Let $s, \tilde{s}$ be (classical) solutions of

$$
\begin{aligned}
\partial_{t} s+\operatorname{div}(f(s) u)-D \Delta(g(s)) & =0, \\
\partial_{t}(g(\tilde{s}))+\operatorname{div}(f(\tilde{s}) u)-D \Delta(g(\tilde{s})) & =e .
\end{aligned}
$$

Then for all nonnegative $\beta, \phi \in C_{0}^{\infty}(\mathbb{R})$

$$
\begin{aligned}
& -\int_{R} \int_{R}\left\|s(t+\tau)-\tilde{s}\left(t-\frac{\tau}{\lambda}\right)\right\| \phi(\tau) d \tau \partial_{t} \beta(t) d t \\
& \leq\|e\|_{L^{1}\left(Q_{T}\right)} \sup _{t \in R} \beta(t) \int_{R} \phi(\tau) d \tau \\
& \quad+\left\{\sup _{t \in(0, T)}\|g(s(t))-s(t)\|+\sup _{t \in(0, T)}\|g(\tilde{s}(t))-\tilde{s}(t)\|\right\} \\
& \quad \times\left\{\int_{R}\left|\partial_{t} \beta(t)\right| d t \int_{R} \phi(\tau) d \tau+\int_{R} \beta(t) d t \int_{R}\left|\partial_{t} \phi(\tau)\right| d \tau\right\}
\end{aligned}
$$

provided that

$$
\gamma\left(t+\tau, t-\frac{\tau}{\lambda}\right)=\beta(t) \phi(\tau)
$$

defines a $\gamma$ with compact support in $(0, T)^{2}$.

Proof. The main idea in proving the $L^{1}$-contraction principle in the case $g=$ id is to multiply the difference of both equations with the $\eta_{\delta}^{\prime}(s-\tilde{s})$, where $\eta_{\delta}$ is a smooth and convex approximation of the modulus function, say,

$$
\eta_{\delta}(z):=\left(z^{2}+\delta^{2}\right)^{\frac{1}{2}}
$$

and to integrate over $Q_{t}$ (see for instance [16]) before doing the $\delta \downarrow 0$ limit. In our case we consider the equations at different fixed times $t_{1}$ resp. $t_{2} \in(0, \infty)$ :

$$
\begin{gathered}
\partial_{t} s\left(t_{1}\right)+\operatorname{div}\left(f\left(s\left(t_{1}\right)\right) u\right)-D \Delta\left(g\left(s\left(t_{1}\right)\right)\right)=0 \\
\partial_{t}(g(\tilde{s}))\left(t_{2}\right)+\operatorname{div}\left(f\left(\tilde{s}\left(t_{2}\right)\right) u\right)-D \Delta\left(g\left(\tilde{s}\left(t_{2}\right)\right)\right)=e\left(t_{2}\right),
\end{gathered}
$$

subtract these identities (using time-independence of $u$ )

$$
\partial_{t} s\left(t_{1}\right)-\partial_{t}(g(\tilde{s}))\left(t_{2}\right)+\operatorname{div}\left[\left(f\left(s\left(t_{1}\right)\right)-f\left(\tilde{s}\left(t_{2}\right)\right)\right) u\right]-D \Delta\left[g\left(s\left(t_{1}\right)\right)-g\left(\tilde{s}\left(t_{2}\right)\right)\right]=-e\left(t_{2}\right)
$$

multiply the difference by

$$
\eta_{\delta}^{\prime}\left(g\left(s\left(t_{1}\right)\right)-g\left(\tilde{s}\left(t_{2}\right)\right)\right) \gamma\left(t_{1}, t_{2}\right)
$$


where $\gamma \in C_{0}^{\infty}\left((0, T)^{2}\right)$ is nonnegative, and integrate over space and both time variables. First, let us consider the time derivative term:

$$
\begin{aligned}
& \lim _{\delta \downarrow 0} \int_{(0, \infty)^{2}} \int_{R^{n}}\left\{\partial_{t} s\left(t_{1}\right)-\partial_{t}(g(\tilde{s}))\left(t_{2}\right)\right\} \eta_{\delta}^{\prime}\left(g\left(s\left(t_{1}\right)\right)-g\left(\tilde{s}\left(t_{2}\right)\right)\right) \gamma\left(t_{1}, t_{2}\right) d\left(t_{1}, t_{2}\right) \\
& =\int_{(0, \infty)^{2}} \int_{R^{n}}\left\{\partial_{t} s\left(t_{1}\right)-\partial_{t}(g(\tilde{s}))\left(t_{2}\right)\right\} \operatorname{sign}\left(g\left(s\left(t_{1}\right)\right)-g\left(\tilde{s}\left(t_{2}\right)\right)\right) \gamma\left(t_{1}, t_{2}\right) d\left(t_{1}, t_{2}\right) \\
& =\int_{(0, \infty)^{2}} \int_{R^{n}}\left\{\partial_{t} s\left(t_{1}\right) \operatorname{sign}\left(s\left(t_{1}\right)-s\left(t_{2}\right)\right)\right. \\
& \left.\quad+\partial_{t}(g(\tilde{s}))\left(t_{2}\right) \operatorname{sign}\left(g\left(\tilde{s}\left(t_{2}\right)\right)-g\left(s\left(t_{1}\right)\right)\right)\right\} \gamma\left(t_{1}, t_{2}\right) d\left(t_{1}, t_{2}\right) \\
& =\lim _{\delta \downarrow 0} \int_{(0, \infty)^{2}} \int_{R^{n}}\left\{\partial_{t_{1}}\left[\eta_{\delta}\left(s\left(t_{1}\right)-\tilde{s}\left(t_{2}\right)\right)\right]\right. \\
& \left.\quad+\partial_{t_{2}}\left[\eta_{\delta}\left(g\left(\tilde{s}\left(t_{2}\right)\right)-g\left(s\left(t_{2}\right)\right)\right)\right]\right\} \gamma\left(t_{1}, t_{2}\right) d\left(t_{1}, t_{2}\right) \\
& =-\lim _{\delta \downarrow 0} \int_{(0, \infty)^{2}} \int_{R^{n}}\left\{\eta_{\delta}\left(s\left(t_{1}\right)-\tilde{s}\left(t_{2}\right)\right) \partial_{t_{1}} \gamma\left(t_{1}, t_{2}\right)\right. \\
& \left.+\eta_{\delta}\left(g\left(\tilde{s}\left(t_{2}\right)\right)-g\left(s\left(t_{2}\right)\right)\right) \partial_{t_{2}} \gamma\left(t_{1}, t_{2}\right)\right\} d\left(t_{1}, t_{2}\right) \\
& =-\int_{(0, \infty)^{2}}\left\{\begin{array}{c}
\quad\left\{s\left(t_{1}\right)-\tilde{s}\left(t_{2}\right) \mid \partial_{t_{1}} \gamma\left(t_{1}, t_{2}\right)\right. \\
\left.+\left|g\left(\tilde{s}\left(t_{2}\right)\right)-g\left(s\left(t_{1}\right)\right)\right| \partial_{t_{2}} \gamma\left(t_{1}, t_{2}\right)\right\} d\left(t_{1}, t_{2}\right) .
\end{array}\right.
\end{aligned}
$$

We will now show that the space derivative term is nonnegative in the limit $\delta \downarrow 0$. Indeed,

$$
\begin{aligned}
\int_{R^{n}}\left\{\operatorname{div}\left[\left(f\left(s\left(t_{1}\right)\right)-f\left(\tilde{s}\left(t_{2}\right)\right)\right) u\right]-D \Delta\left[g\left(s\left(t_{1}\right)\right)-g\left(\tilde{s}\left(t_{2}\right)\right)\right]\right\} \cdot \eta_{\delta}^{\prime}\left(g\left(s\left(t_{1}\right)\right)-g\left(\tilde{s}\left(t_{2}\right)\right)\right) \\
=\int_{R^{n}} \eta_{\delta}^{\prime \prime}\left(g\left(s\left(t_{1}\right)\right)-g\left(\tilde{s}\left(t_{2}\right)\right)\right) \nabla\left[g\left(s\left(t_{1}\right)\right)-g\left(\tilde{s}\left(t_{2}\right)\right)\right] \\
\quad \times\left\{D \nabla\left[g\left(s\left(t_{1}\right)\right)-g\left(\tilde{s}\left(t_{2}\right)\right)\right]-\left(f\left(s\left(t_{1}\right)\right)-f\left(\tilde{s}\left(t_{2}\right)\right)\right) u\right\} \\
\geq-\frac{1}{4 D} \int_{R^{n}} \eta_{\delta}^{\prime \prime}\left(g\left(s\left(t_{1}\right)\right)-g\left(\tilde{s}\left(t_{2}\right)\right)\right)\left|f\left(s\left(t_{1}\right)\right)-f\left(\tilde{s}\left(t_{2}\right)\right)\right|^{2}|u|^{2} \\
\geq-\frac{C}{4 D} \int_{R^{n}} \eta_{\delta}^{\prime \prime}\left(g\left(s\left(t_{1}\right)\right)-g\left(\tilde{s}\left(t_{2}\right)\right)\right)\left|g\left(s\left(t_{1}\right)\right)-g\left(\tilde{s}\left(t_{2}\right)\right)\right|^{2}|u|^{2} \\
\geq-\frac{C \delta}{4 D} \int_{R^{n}}|u|^{2}
\end{aligned}
$$

where $C$ denotes the Lipschitz constant of $f \circ g^{-1}$ on the bounded range of $s$ and $\tilde{s}$ and we have used

$$
0 \leq \eta_{\delta}^{\prime \prime}(z)|z|^{2} \leq \delta
$$


So we obtain

$$
\begin{aligned}
-\int_{(0, \infty)^{2}}\left\{\left\|s\left(t_{1}\right)-\tilde{s}\left(t_{2}\right)\right\| \partial_{t_{1}} \gamma\left(t_{1}, t_{2}\right)\right. & \left.+\left\|g\left(\tilde{s}\left(t_{2}\right)\right)-g\left(s\left(t_{1}\right)\right)\right\| \partial_{t_{2}} \gamma\left(t_{1}, t_{2}\right)\right\} d\left(t_{1}, t_{2}\right) \\
& \leq \int_{(0, \infty)^{2}}\left\|e\left(t_{2}\right)\right\| \gamma\left(t_{1}, t_{2}\right) d\left(t_{1}, t_{2}\right)
\end{aligned}
$$

We introduce new variables $(t, \tau)$ according to

$$
\left(\begin{array}{l}
t_{1} \\
t_{2}
\end{array}\right)=\left(\begin{array}{cc}
1 & 1 \\
1 & -\frac{1}{\lambda}
\end{array}\right)\left(\begin{array}{l}
t \\
\tau
\end{array}\right) .
$$

Then

$$
\begin{aligned}
d\left(t_{1}, t_{2}\right) & =\frac{1+\lambda}{\lambda} d(t, \tau) \\
\left(\phi \partial_{t} \beta, \beta \partial_{\tau} \phi\right) & =\left(\left(\partial_{t_{1}}+\partial_{t_{2}}\right) \gamma,\left(\partial_{t_{1}}-\frac{1}{\lambda} \partial_{t_{2}}\right) \gamma\right)
\end{aligned}
$$

for $\gamma$ given by

$$
\gamma\left(t+\tau, t-\frac{\tau}{\lambda}\right)=\beta(t) \phi(\tau)
$$

Rewriting the integrand of the left-hand side of (13) in the following way,

$$
\begin{gathered}
\left\|s\left(t_{1}\right)-\tilde{s}\left(t_{2}\right)\right\| \partial_{t_{1}} \gamma\left(t_{1}, t_{2}\right)+\left\|g\left(\tilde{s}\left(t_{2}\right)\right)-g\left(s\left(t_{1}\right)\right)\right\| \partial_{t_{2}} \gamma\left(t_{1}, t_{2}\right) \\
=\left\|s\left(t_{1}\right)-\tilde{s}\left(t_{2}\right)\right\|\left(\partial_{t_{1}}+\partial_{t_{2}}\right) \gamma\left(t_{1}, t_{2}\right) \\
\quad+\frac{\lambda}{1+\lambda}\left\{\left\|s\left(t_{1}\right)-\tilde{s}\left(t_{2}\right)\right\|-\left\|g\left(\tilde{s}\left(t_{2}\right)\right)-g\left(s\left(t_{1}\right)\right)\right\|\right\} \\
\quad \times\left[-\left(\partial_{t_{1}}+\partial_{t_{2}}\right)+\left(\partial_{t_{1}}-\frac{1}{\lambda} \partial_{t_{2}}\right)\right] \gamma\left(t_{1}, t_{2}\right)
\end{gathered}
$$

we easily obtain the statement of Lemma 2 .

We now apply Lemma 2 to $s_{D}^{*}$ and $\tilde{s}_{D}(t)=\Phi\left(s_{D}(\lambda t)\right)$ and use estimate (12) to obtain

$$
\begin{aligned}
\int_{R} \int_{R}\left\|s_{D}^{*}(t+\tau)-s_{D}(\lambda t-\tau)\right\| \phi(\tau) d \tau \partial_{t} \beta(t) d t \\
\leq E_{1} K_{\lambda T} \sup _{t \in R} \beta(t) \int_{R} \phi(\tau) d \tau \\
+E_{2} K_{\lambda T} \int_{R}\left|\partial_{t} \beta(t)\right| d t \int_{R} \phi(\tau) d \tau \\
+E_{3} K_{\lambda T}\left\{\int_{R}\left|\partial_{t} \beta(t)\right| d t \int_{R} \phi(\tau) d \tau+\int_{R} \beta(t) d t \int_{R}\left|\partial_{\tau} \phi(\tau)\right| d \tau\right\},
\end{aligned}
$$

where

$$
\begin{aligned}
& E_{1}:=\int_{R}\left|(g \circ \phi)^{\prime \prime}(z)\right|(1+2|f(z)|) d z \\
& E_{2}:=\sup _{z \in R}\left|\frac{1}{z}(\Phi(z)-z)\right| \\
& E_{3}:=\sup _{z \in R}\left|\frac{1}{z}(g(z)-z)\right|+\sup _{z \in R}\left|\frac{1}{z}(g(\Phi(z))-\Phi(z))\right|, \\
& K_{t}:=\left\|s^{0}\right\|+t\|\operatorname{div} u\| .
\end{aligned}
$$


Here we made implicit use of the $L^{1}$-contraction principle for Eqs. (10) and (11) yielding $\left\|s_{D}(t)\right\|,\left\|s_{D}^{*}(t)\right\| \leq\left\|s^{0}\right\|$. We let $D$ tend to zero and choose (formally)

$$
\begin{aligned}
& \beta(t)=\left\{\begin{array}{ll}
1 & \text { for } t^{-}<t<t^{+} \\
0 & \text { else }
\end{array}\right\}, \\
& \phi(\tau)=\phi_{\varepsilon}(\tau):=\frac{1}{\varepsilon} \phi_{1}\left(\frac{\tau}{\varepsilon}\right),
\end{aligned}
$$

where

$$
\phi_{1}(\tau):=\left\{\begin{array}{ll}
2 & \text { for }-\frac{1}{2}<\tau<0 \\
0 & \text { else }
\end{array}\right\}
$$

for some $\varepsilon>0$ and $\frac{\varepsilon}{2}<t^{-}<t^{+}$. We end up with

$$
\begin{aligned}
\int_{R}\left\|s\left(t^{+}+\tau\right)-s\left(\lambda t^{+}-\tau\right)\right\| \phi_{\varepsilon}(\tau) d \tau \\
\leq \int_{R}\left\|s\left(t^{-}+\tau\right)-s\left(\lambda t^{-}-\tau\right)\right\| \phi_{\varepsilon}(\tau) d \tau \\
\quad+\left(E_{1}+2 E_{2}+2 E_{3}+\frac{4}{\varepsilon}\left(t^{+}-t^{-}\right) E_{3}\right) K_{\lambda t^{+}+\varepsilon}
\end{aligned}
$$

The averaging in $\tau$ of fixed order $\varepsilon$ prevents us from making use of the fact that

$$
\lim _{t \downarrow 0}\|s(t)-s(\lambda t)\|=0
$$

by choosing $t^{-}=0$. Our strategy is to imitate the appropriate scaling $\varepsilon \sim t$ with help of some iteration procedure (Lemma 4). It turns out that the latter relies on the more specific scaling $\varepsilon \sim(1-\lambda) t^{+}$. So in view of $(15)$, we have to minimize - and thereby balance - the error terms

$$
E_{1}+E_{2}+E_{3} \text { and } \quad \frac{1}{1-\lambda} E_{3}
$$

by appropriately constructing $\Phi$ (Lemma 3 ). Observe that this problem is one of real variable calculus and is decoupled from Eq. (1).

Lemma 3. There exist diffeomorphisms $\Phi, g: \mathbb{R} \rightarrow \mathbb{R}$ such that

$$
\begin{gathered}
\Phi^{\prime \prime} \text { has compact support and } \Phi(0)=0, \\
g^{\prime}-1 \text { has compact support and } g(0)=0, \\
f^{\prime}(\phi(z))=\lambda g^{\prime}(\Phi(z)) f^{\prime}(z),
\end{gathered}
$$

which are estimated as follows:

$$
\max \left\{E_{1}, E_{2}, \frac{1}{1-\lambda} E_{3}\right\} \leq C(1-\lambda)^{\alpha},
$$

where $0<\alpha, C<\infty$ only depend on $f$.

Proof. Let $C<\infty$ denote a generic constant only depending on $f$. Without loss of generality we may assume that $\delta:=1-\lambda$ is sufficiently small. By definition of strictly nonlinear, there exist finitely many $w_{1}<\cdots<w_{M}$ such that

$$
\left\{w_{1}, \ldots, w_{M}\right\}=\left\{z \in \mathbb{R} \mid f^{\prime \prime}(z)=0\right\} .
$$


Let us start by assuming that, additionally,

A) there exists an $h>0$ such that for each $j \in\{1, \ldots, M\}$,

$$
f^{\prime}\left(w_{j}+z\right)-f^{\prime}\left(w_{j}\right) \text { for } z \in(-h, h)
$$

is represented by a homogeneous polynomial in $z$.

Define $\Phi_{*}$ to be the function with maximal domain $D \subset\left(-\infty, w_{1}\right) \cup \cdots \cup\left(w_{M},+\infty\right)$ such that

$$
\begin{array}{ccc}
\Phi_{*}\left(\left(-\infty, w_{1}\right) \cap D\right) & \subset\left(-\infty, w_{1}\right) \\
& \vdots & \\
\Phi_{*}\left(\left(w_{M},+\infty\right) \cap D\right) & \subset & \left(w_{M},+\infty\right)
\end{array}
$$

and

$$
f^{\prime}\left(\Phi_{*}(z)\right)=\lambda f^{\prime}(z) \text { for all } z \in D .
$$

Let $z_{1}^{0}<\cdots<z_{N}^{0}$ denote those points among $w_{1}, \ldots, w_{M}$ that are not zeros of $f^{\prime}$, i.e.,

$$
\left\{z_{1}^{0}, \ldots, z_{N}^{0}\right\}=\left\{z \in \mathbb{R} \mid f^{\prime}(z) \neq 0 \text { and } f^{\prime \prime}(z)=0\right\} .
$$

We contend that $\Phi_{*}$ is well behaved on

$$
B:=\mathbb{R}-\bigcup_{i=1}^{N}\left[z_{i}^{0}-\frac{h}{2}, z_{i}^{0}+\frac{h}{2}\right] .
$$

Obviously, troubles only can arise at the $w_{j}$ 's or at infinity. Consider first a $w_{j}$ lying in $B$, i.e., $f^{\prime}\left(w_{j}\right)=f^{\prime \prime}\left(w_{j}\right)=0$. Without loss of generality we may assume that $w_{j}=0$. Thanks to $\mathrm{A}$, there exist an integer $m$ and $a \neq 0$ such that

$$
f^{\prime}(z)=a z^{m} \quad \text { for } z \in(-h, h)
$$

hence $(-h, h) \subset D$ and

$$
\Phi_{*}(z)=\lambda^{\frac{1}{m}} z \quad \text { for } z \in(-h, h) .
$$

On the other hand we have, owing to part 2 of the definition of "strictly nonlinear", that $\left(-\infty, \lambda^{\frac{1}{p_{-}}} M\right) \cup\left(\lambda^{\frac{1}{p_{+}}} M,+\infty\right) \subset D$ and

$$
\Phi_{*}(z)=\left\{\begin{array}{ll}
\lambda^{\frac{1}{p_{+}}} z & \text { for } z>\lambda^{-\frac{1}{p_{+}}} M \\
\lambda^{\frac{1}{p_{-}}} z & \text { for } z<\lambda^{-\frac{1}{p_{-}}} M
\end{array}\right\}
$$

- so there is no problem at infinity either. We gather

$$
\int_{B}\left|\Phi_{*}^{\prime \prime}\right| \leq C \delta \quad \text { and } \quad \sup _{B}\left|\Phi_{*}^{\prime}-1\right| \leq C \delta
$$

Let us now investigate the behaviour of $\Phi_{*}$ on $\left[z_{i}^{0}-\frac{h}{2}, z_{i}^{0}+\frac{h}{2}\right] \cap D$. Without loss of generality we may suppose $z_{i}^{0}=0$ and omit the $i$ indices. As a consequence of $\mathrm{A}$, there exist an integer $m$ and $a, b \neq 0$ such that

$$
f^{\prime}(z)=a z^{m}+b \quad \text { for } z \in(-h, h) .
$$


It is most convenient to investigate the one-sided neighborhoods $[-h, 0],[0, h]$ and the cases $\frac{b}{a}>0, \frac{b}{a}<0$ separately. Let us for instance consider $[0, h]$ in the case $\frac{b}{a}>0$ : In this situation, $\Phi_{*}$ is defined on $\left[\left(\frac{b}{a} \frac{\delta}{\lambda}\right)^{1 / m}, \frac{h}{2}\right]$ and satisfies

$$
\begin{aligned}
& \Phi_{*}(z)=\lambda^{\frac{1}{m}}\left(z^{m}-\frac{b}{a} \frac{\delta}{\lambda}\right)^{\frac{1}{m}}, \\
& \Phi_{*}^{\prime}(z)=\lambda^{\frac{1}{m}}\left[\frac{z}{\left(z^{m}-\frac{b}{a} \frac{\delta}{\lambda}\right)^{\frac{1}{m}}}\right]^{m-1}, \\
& \Phi_{*}^{\prime \prime}(z) \leq 0 .
\end{aligned}
$$

We now state at which rate the quantities of interest diverge at the singularity: For small $\rho \geq\left(2 \frac{b}{a} \frac{\delta}{\lambda}\right)^{1 / m}$ we obtain the following estimates:

$$
\begin{gathered}
\int_{\left(\rho, \frac{h}{2}\right)}\left|\Phi_{*}^{\prime \prime}\right| \leq \Phi_{*}^{\prime}(\rho)-\lambda^{\frac{1}{m}} \leq C \frac{\delta}{\rho^{m}}, \\
\sup _{\left(\rho, \frac{h}{2}\right)}\left|\Phi_{*}^{\prime}-1\right| \leq\left(\Phi_{*}^{\prime}(\rho)-\lambda^{\frac{1}{m}}\right)+\left(1-\lambda^{\frac{1}{m}}\right) \leq C \frac{\delta}{\rho^{m}}, \\
\left|\frac{\Phi_{*}(\rho)}{\rho}-1\right| \leq C \frac{\delta}{\rho^{m}} \quad \text { and } \quad \Phi_{*}(\rho) \leq C \rho .
\end{gathered}
$$

We define $\Phi$ on $\left[0, \frac{h}{2}\right]$ by

$$
\Phi(z):=\left\{\begin{array}{ll}
\Phi_{*}(z) & \text { for } z \in\left[\rho, \frac{h}{2}\right] \\
\Phi_{*}(\rho) \frac{z}{\rho} & \text { for } z \in[0, \rho]
\end{array}\right\},
$$

where $\rho \geq\left(2 \frac{a}{b} \frac{\delta}{\lambda}\right)^{1 / m}$ is a function of $\delta$-yet to be chosen. The above estimates for $\Phi_{*}$ translate into the ones below for $\Phi$ :

$$
\begin{array}{r}
\operatorname{TV}\left(\Phi^{\prime \prime},\left(0, \frac{h}{2}\right)\right)=\left|\frac{\Phi_{*}(\rho)}{\rho}-\Phi_{*}^{\prime}(\rho)\right|+\int_{\left(\rho, \frac{h}{2}\right)}\left|\Phi_{*}^{\prime \prime}\right| \leq C \frac{\delta}{\rho^{m}}, \\
\operatorname{ess} \sup _{\left(0, \frac{h}{2}\right)}\left|\Phi^{\prime}-1\right|=\max \left\{\left|\frac{\Phi_{*}(\rho)}{\rho}-1\right|, \sup _{\left(\rho, \frac{h}{2}\right)}\left|\Phi^{\prime}-1\right|\right\} \leq C \frac{\delta}{\rho^{m}} .
\end{array}
$$

In order to meet (17), $g$ has to satisfy

$$
g^{\prime}(\Phi(z))=\frac{f^{\prime}(\Phi(z))}{\lambda f^{\prime}(z)}=\left\{\begin{array}{ll}
1+\frac{\delta}{\lambda} \frac{1-\left(\frac{z}{\rho}\right)^{m}}{\frac{a}{b} z^{m}+1} & \text { for } z \in(0, \rho] \\
1 & \text { for } z \in\left[\rho, \frac{h}{2}\right)
\end{array}\right\} .
$$

Thus

$$
\begin{gathered}
\operatorname{TV}\left(g^{\prime}\left(0, \frac{h}{2}\right)\right)=\frac{1}{\lambda}-1 \leq C \delta, \\
\sup _{\Phi\left(\left(0, \frac{h}{2}\right)\right)}\left|g^{\prime}-1\right|=\frac{1}{\lambda}-1 \leq C \delta, \\
\mathcal{L}^{1}\left(\left\{g^{\prime} \neq 1\right\} \cap \Phi\left(\left(0, \frac{h}{2}\right)\right)\right) \leq C \delta .
\end{gathered}
$$

Fixing $\rho=\rho(\delta)$ to be

$$
\rho=\delta^{\alpha} \quad \text { where } \alpha:=\frac{1}{m+1},
$$


the estimates turn into

$$
\begin{aligned}
\operatorname{TV}\left(\Phi^{\prime \prime},\left(0, \frac{h}{2}\right)\right) & \leq C \delta^{\alpha}, \\
\sup _{\left(0, \frac{h}{2}\right)}\left|\Phi^{\prime}-1\right| & \leq C \delta^{\alpha}, \\
\operatorname{TV}\left(g^{\prime}, \Phi\left(\left(0, \frac{h}{2}\right)\right)\right) & \leq C \delta, \\
\sup _{\Phi\left(\left(0, \frac{h}{2}\right)\right)}\left|g^{\prime}-1\right| & \leq C \delta, \\
\mathcal{L}^{1}\left(\left\{g^{\prime} \neq 1\right\} \cap \Phi\left(\left(0, \frac{h}{2}\right)\right)\right) & \leq C \delta^{\alpha} .
\end{aligned}
$$

Looking at the partial results (18) and (20), we realize that we have constructed two diffeomorphisms $\Phi, g: \mathbb{R} \rightarrow \mathbb{R}$, the properties (16) and (17) being estimated as

$$
\begin{aligned}
\operatorname{TV}\left(\Phi^{\prime \prime}\right) & \leq C \delta^{\alpha}, \\
\sup \left|\Phi^{\prime}-1\right| & \leq C \delta^{\alpha}, \\
\operatorname{TV}\left(g^{\prime}\right) & \leq C \delta, \\
\sup \left|g^{\prime}-1\right| & \leq C \delta, \\
\mathcal{L}^{1}\left(\left\{g^{\prime} \neq 1\right\}\right) & \leq C \delta^{\alpha}, \quad g^{\prime}=1 \text { on }(-h, h),
\end{aligned}
$$

where $\alpha:=\frac{1}{m+1}$ and $m$ now is the maximum of the $m$ 's occurring in the representations (19) in the neighborhood of the $z_{i}^{0}$ 's. The last statement of (21) is a consequence of $0 \notin\left\{z_{1}^{0}, \ldots, z_{N}^{0}\right\}$, since $f^{\prime}(0)=0$ by assumption.

Now we will show how to eliminate the additional assumption A. By definition of "strictly nonlinear", we can construct a diffeomorphism $\Psi: \mathbb{R} \rightarrow \mathbb{R}$ with

$$
\Psi^{\prime \prime} \text { has compact support and } \Psi(0)=0
$$

such that $\tilde{f}$ defined by

$$
\tilde{f}^{\prime}(z)=f^{\prime}(\Psi(z)) \text { and } \quad \tilde{f}(0)=0
$$

complies with A. Let $\widetilde{\Phi}$ and $\tilde{g}$ be the above constructions with respect to $\tilde{f}$. Define the diffeomorphisms $\Phi, g: \mathbb{R} \rightarrow \mathbb{R}$ by

$$
\begin{gathered}
\Phi:=\Psi \circ \widetilde{\Phi} \circ \Psi^{-1} \\
\tilde{g}^{\prime}(z)=g^{\prime}(\Psi(z)) \quad \text { and } \quad g(0)=0 .
\end{gathered}
$$

Obviously, $\Phi$ and $g$ obey (16) and by construction also (17). Straightforward calculations starting from

$$
\begin{aligned}
\Phi^{\prime}(\Psi(z)) & =\frac{\Psi^{\prime}(\widetilde{\Phi}(z))}{\Psi^{\prime}(z)} \widetilde{\Phi}^{\prime}(z) \\
g^{\prime}(\Psi(z)) & =\tilde{g}^{\prime}(z)
\end{aligned}
$$

reveal that (21) carries on as well. 
It remains to show that $E_{1}, E_{2}$, and $E_{3}$ can be estimated with the help of $(21)$. This is indeed the case:

$$
\begin{array}{rlr}
E_{1} & \leq C \operatorname{TV}\left((g \circ \Phi)^{\prime}\right) & \\
& \leq C\left\{\sup g^{\prime} \operatorname{TV}\left(\Phi^{\prime}\right)+\sup \Phi^{\prime} \operatorname{TV}\left(g^{\prime}\right)\right\} & \leq C \delta^{\alpha}, \\
E_{2} & \leq \sup \left|\Phi^{\prime}-1\right| & \leq C \delta^{\alpha}, \\
E_{3} & \leq\left(1+\sup _{z \in R}\left|\frac{\phi(z)}{z}\right|\right) \sup _{z \in R}\left|\frac{1}{z}(g(z)-z)\right| & \\
& \leq C\left(1+\sup \Phi^{\prime}\right) \sup \left|g^{\prime}-1\right| \mathcal{L}^{1}\left(\left\{g^{\prime} \neq 1\right\}\right) \leq C \delta^{1+\alpha}
\end{array}
$$

(in the $E_{1}$-estimate we have used that $(g \circ \Phi)^{\prime \prime}$ has compact support, whereas in the $E_{3}$-estimate we brought into play that $g^{\prime}=1$ in a neighborhood of 0 , see (21)). Here $\alpha:=\frac{1}{m+1}$ where $m$ is now given by

$m:=\max \left\{k \geq 2 \mid\right.$ there exists a $z_{0} \in \mathbb{R}$ such that

$$
\left.f^{\prime}\left(z_{0}\right) \neq 0, f^{\prime \prime}\left(z_{0}\right)=\cdots=f^{(k)}\left(z_{0}\right)=0, f^{(k+1)}\left(z_{0}\right) \neq 0\right\} .
$$

We apply Lemma 3 to (15) and obtain

$$
\begin{aligned}
& \int_{R}\left\|s\left(t^{+}+\tau\right)-s\left(\lambda t^{+}-\tau\right)\right\| \phi_{\varepsilon}(\tau) d \tau \\
& \leq \int_{R}\left\|s\left(t^{-}+\tau\right)-s\left(\lambda t^{-}-\tau\right)\right\| \phi_{\varepsilon}(\tau) d \tau \\
& \quad+C\left(1+\frac{(1-\lambda)\left(t^{+}-t^{-}\right)}{\varepsilon}\right)(1-\lambda)^{\alpha} K_{\lambda t^{+}+\varepsilon}
\end{aligned}
$$

for all $\varepsilon>0$ and $\frac{\varepsilon}{2}>t^{-}>t^{+}$. As announced, the next lemma will estimate the scale invariant entity

$$
\int_{R}\|s(t+\tau)-s(\lambda t-\tau)\| \phi_{(1-\lambda) t}(\tau) d \tau
$$

by an iteration procedure in $t$ (for the very special choice of $\phi_{\varepsilon}$ in (14)), only using (22) and

$$
\lim _{t \downarrow 0}\left\|s(t)-s^{0}\right\|=0 .
$$

LEMMA 4. For all $t>0$, the estimate

$$
\int_{R}\|s(t+\tau)-s(\lambda t-\tau)\| \phi_{(1-\lambda) t}(\tau) d \tau \leq C K_{t}(1-\lambda)^{\alpha}
$$

holds.

Proof. We set for convenience $\delta=1-\lambda$ and

$$
\begin{aligned}
g_{\delta}(t) & :=\int_{R}\|s(t+\tau)-s(\lambda t-\tau)\| \phi_{\delta t}(\tau) d \tau \\
& =\frac{2}{\delta t} \int_{-\frac{\delta t}{2}}^{0}\|s(t+\tau)-s(\lambda t-\tau)\| d \tau
\end{aligned}
$$


The main step consists in proving the estimate

$$
g_{\delta}(t) \leq \frac{1}{2}\left(g_{\delta_{0}}\left(t_{0}\right)+C K_{t} \delta_{0}^{\alpha}\right)+\frac{1}{2}\left(g_{\delta_{1}}\left(t_{1}\right)+C K_{t} \delta_{1}^{\alpha}\right),
$$

where

$$
\left(t_{0}, \delta_{0}\right):=\left(\frac{1}{2} t, \delta\right), \quad\left(t_{1}, \delta_{1}\right):=\left(\frac{1}{2}\left(1-\frac{\delta}{4}\right) t, \frac{1}{2}\left(1-\frac{\delta}{4}\right)^{-1} \delta\right) \sim\left(\frac{1}{2} t, \frac{1}{2} \delta\right) .
$$

Observe that

$$
\begin{aligned}
& \int_{R}\|s(t+\tau)-s((1-\delta) t-\tau)\| \phi_{\delta t}(\tau) d \tau \\
& =\frac{1}{2} \int_{R}\left\|s(t+\tau)-s\left(\left(1-\delta_{0}\right) t-\tau\right)\right\| \phi_{\frac{\delta t}{2}}(\tau) d \tau \\
& \quad+\frac{1}{2} \int_{R}\left\|s\left(\left(1-\frac{\delta}{4}\right) t+\tau\right)-s\left(\left(1-\delta_{1}\right)\left(1-\frac{\delta}{4}\right) t-\tau\right)\right\| \phi_{\frac{\delta t}{2}}(\tau) d \tau .
\end{aligned}
$$

Apply (22) to the right-hand side integrals separately:

$$
\begin{aligned}
& \int_{R}\left\|s(t+\tau)-s\left(\left(1-\delta_{0}\right) t-\tau\right)\right\| \phi_{\frac{\delta t}{2}}(\tau) d \tau \\
& \quad \leq \int_{R}\left\|s\left(t_{0}+\tau\right)-s\left(\left(1-\delta_{0}\right) t_{0}-\tau\right)\right\| \phi_{\frac{\delta t}{2}}(\tau) d \tau+C K_{t} \delta_{0}^{\alpha}, \\
& \int_{R}\left\|s\left(\left(1-\frac{\delta}{4}\right) t+\tau\right)-s\left(\left(1-\delta_{1}\right)\left(1-\frac{\delta}{4}\right) t-\tau\right)\right\| \phi_{\frac{\delta t}{2}}(\tau) d \tau \\
& \quad \leq \int_{R}\left\|s\left(t_{1}+\tau\right)-s\left(\left(1-\delta_{1}\right) t_{1}-\tau\right)\right\| \phi_{\frac{\delta t}{2}}(\tau) d \tau+C K_{t} \delta_{1}^{\alpha} .
\end{aligned}
$$

Notice that the right-hand side integrals can be written as

$$
\begin{aligned}
\int_{R} \| & s\left(t_{0}+\tau\right)-s\left(\left(1-\delta_{0}\right) t_{0}-\tau\right) \| \phi_{\frac{\delta t}{2}}(\tau) d \tau \\
= & \int_{R}\left\|s\left(t_{0}+\tau\right)-s\left(\left(1-\delta_{0}\right) t_{0}-\tau\right)\right\| \phi_{\delta_{0} t_{0}}(\tau) d \tau
\end{aligned}
$$

and

$$
\begin{aligned}
\int_{R}\left\|s\left(t_{1}+\tau\right)-s\left(\left(1-\delta_{1}\right) t_{1}-\tau\right)\right\| \phi_{\frac{\delta t}{2}}(\tau) d \tau \\
=\int_{R}\left\|s\left(t_{1}+\tau\right)-s\left(\left(1-\delta_{1}\right) t_{1}-\tau\right)\right\| \phi_{\frac{\delta t}{4}}(\tau) d \tau \\
=\int_{R}\left\|s\left(t_{1}+\tau\right)-s\left(\left(1-\delta_{1}\right) t_{1}-\tau\right)\right\| \phi_{\delta_{1} t_{1}}(\tau) d \tau
\end{aligned}
$$

This establishes (25).

We will now iterate (25). Fix initial $\delta \in(0,1)$ and $t>0$. Define

$$
\left(t_{\beta}, \delta_{\beta}\right) \text { for } \beta \in\{0,1\}^{k} \text { and } k \in\{1,2, \ldots\}
$$

inductively in $k$ by

$$
\begin{aligned}
\left(t_{0}, \delta_{0}\right) & =\left(\frac{1}{2} t, \delta\right) & \left(t_{1}, \delta_{1}\right) & =\left(\frac{1}{2}\left(1-\frac{\delta}{4}\right) t, \frac{1}{2}\left(1-\frac{\delta}{4}\right)^{-1} \delta\right), \\
\left(t_{\beta 0}, \delta_{\beta 0}\right) & =\left(\frac{1}{2} t_{\beta}, \delta_{\beta}\right), & \left(t_{\beta 1}, \delta_{\beta 1}\right) & =\left(\frac{1}{2}\left(1-\frac{\delta}{4}\right) t_{\beta}, \frac{1}{2}\left(1-\frac{\delta}{4}\right)^{-1} \delta_{\beta}\right) .
\end{aligned}
$$


From (25) we deduce by induction in $N$

$$
g_{\delta}(t) \leq \sum_{\beta \in\{0,1\}^{N}} 2^{-N} g_{\delta_{\beta}}\left(t_{\beta}\right)+C K_{t} \sum_{k=1}^{N} \sum_{\beta \in\{0,1\}^{k}} 2^{-k} \delta_{\beta}^{\alpha}
$$

Also by induction in $k$,

$$
t_{\beta} \leq\left(\frac{1}{2}\right)^{k} t, \quad \delta_{\beta} \leq\left(\frac{2}{3}\right)^{|\beta|} \delta \quad \text { for } \beta \in\{0,1\}^{k} \text { and } k \in\{1,2, \ldots\}
$$

We conclude

$$
g_{\delta}(t) \leq \sup _{\substack{0<t<\left(\frac{1}{2}\right)^{N} \\ 0<\tilde{\delta}<\delta}} g_{\tilde{\delta}}(\tilde{t})+C K_{t} \delta^{\alpha} \sum_{k=1}^{N} \sum_{\beta \in\{0,1\}^{k}} 2^{-k}\left(\frac{2}{3}\right)^{\alpha|\beta|}
$$

Because of $\lim _{\tilde{t} \downarrow 0} g_{\tilde{\delta}}(\tilde{t})=0$ uniformly in $\tilde{\delta}$ (here we use (23)) and

$$
\begin{aligned}
\sum_{k=1}^{N} \sum_{\beta \in\{0,1\}^{k}} 2^{-k}\left(\frac{2}{3}\right)^{\alpha|\beta|} & =\sum_{k=1}^{N} \sum_{\beta \in\{0,1\}^{k}}\left(\frac{1}{2}\right)^{k-|\beta|}\left(\frac{1}{2}\left(\frac{2}{3}\right)^{\alpha}\right)^{|\beta|} \\
& =\sum_{k=1}^{N}\left(\frac{1}{2}+\frac{1}{2}\left(\frac{2}{3}\right)^{\alpha}\right)^{k} \\
& \leq \frac{1+\left(\frac{2}{3}\right)^{\alpha}}{1-\left(\frac{2}{3}\right)^{\alpha}}
\end{aligned}
$$

we obtain $g_{\delta}(t) \leq C K_{t} \delta^{\alpha}$ in the limit $N \uparrow \infty$.

The last step consists in deriving a pointwise (in $t$ ) estimate from the averaged estimate stated in Lemma 4. All we need is $(24)$ and $s \in C^{0}\left([0, \infty), L^{1}\left(\mathbb{R}^{n}\right)\right)$. This last lemma is inspired by the Campanato technique of proving Hölder continuity for solutions of elliptic equations (see, for instance, [2]).

LEMMA 5. For all $t>0$,

$$
\|s(t)-s(\lambda t)\| \leq C K_{t}(1-\lambda)^{\alpha}
$$

Proof. Without loss of generality we may assume $\delta:=1-\lambda \in\left(0, \frac{1}{2}\right)$. Let us first argue that

$$
\frac{1}{\delta t} \int_{-\delta t}^{0}\|s(t+\tau)-s((1-\delta) t+\tau)\| d \tau \leq C K_{t} \delta^{\alpha}
$$


Indeed, the left-hand side can be written and estimated as follows:

$$
\begin{aligned}
& \frac{1}{\delta t} \int_{-\delta t / 2}^{0}\|s(t+\tau)-s((1-\delta) t+\tau)\| d \tau+\frac{1}{\delta t} \int_{-\delta t}^{-\delta t / 2}\|s(t+\tau)-s((1-\delta) t+\tau)\| d \tau \\
& \leq \frac{1}{\delta t} \int_{-\delta t / 2}^{0}\|s(t+\tau)-s((1-\delta) t+\tau)\| d \tau \\
& +\frac{1}{\delta t} \int_{-\delta t / 2}^{0}\|s((1-\delta) t-\tau)-s((1-\delta) t+\tau)\| d \tau \\
& +\frac{1}{\delta t} \int_{-\delta t}^{-\delta t / 2}\|s(t+\tau)-s((1-2 \delta) t+\tau)\| d \tau \\
& +\frac{1}{\delta t} \int_{-\delta t}^{-\delta t / 2}\|s((1-2 \delta) t+\tau)-s((1-\delta) t+\tau)\| d \tau \\
& =\frac{1}{2} \int_{R}\|s(t+\tau)-s((1-\delta) t-\tau)\| \phi_{\delta t}(\tau) d \tau \\
& +\frac{1}{2} \int_{R}\left\|s\left(t_{1}+\tau\right)-s\left(\left(1-\delta_{1}\right) t_{1}-\tau\right)\right\| \phi_{\delta_{1} t_{1}}(\tau) d \tau \\
& +\frac{1}{2} \int_{R}\left\|s\left(t_{1}+\tau\right)-s\left(\left(1-\delta_{1}\right) t_{1}-\tau\right)\right\| \phi_{\delta_{1} t_{1}}(\tau) d \tau \\
& +\frac{1}{2} \int_{R}\left\|s\left(t_{2}+\tau\right)-s\left(\left(1-\delta_{2}\right) t_{2}-\tau\right)\right\| \phi_{\delta_{2} t_{2}}(\tau) d \tau \\
& \stackrel{(24)}{\leq} C\left\{\frac{1}{2} K_{t} \delta^{\alpha}+K_{t_{1}} \delta_{1}^{\alpha}+\frac{1}{2} K_{t_{2}} \delta_{2}^{\alpha}\right\} \leq C K_{t} \delta^{\alpha},
\end{aligned}
$$

where

$$
\left(t_{1}, \delta_{1}\right):=\left(\left(1-\frac{\delta}{2}\right) t,\left(1-\frac{\delta}{2}\right)^{-1} \delta\right), \quad\left(t_{2}, \delta_{2}\right):=\left((1-\delta) t,(1-\delta)^{-1} \delta\right)
$$

The next step consists in establishing

$$
\frac{1}{\delta t} \int_{0}^{\delta t}\|s(t)-s(t-\tau)\| d \tau \leq C K_{t} \delta^{\alpha}
$$

We do this by iterating the estimate

$$
\frac{1}{\delta t} \int_{0}^{\delta t}\|s(t)-s(t-\tau)\| d \tau \leq C K_{t} \delta^{\alpha}+\frac{1}{\delta t / 2} \int_{0}^{\delta t / 2}\|s(t)-s(t-\tau)\| d \tau
$$


which is obtained in the following way:

$$
\begin{aligned}
\frac{1}{\delta t} \int_{0}^{\delta t} & \|s(t)-s(t-\tau)\| d \tau \\
= & \frac{1}{\delta t} \int_{0}^{\delta t / 2}\|s(t)-s(t-\tau)\| d \tau+\frac{1}{\delta t} \int_{\delta t / 2}^{\delta t}\|s(t)-s(t-\tau)\| d \tau \\
\leq & \frac{1}{\delta t} \int_{0}^{\delta t / 2}\|s(t)-s(t-\tau)\| d \tau+\frac{1}{\delta t} \int_{\delta t / 2}^{\delta t}\left\|s(t)-s\left(\left(1+\frac{\delta}{2}\right) t-\tau\right)\right\| d \tau \\
& +\frac{1}{\delta t} \int_{\delta t / 2}^{\delta t}\left\|s\left(\left(1+\frac{\delta}{2}\right) t-\tau\right)-s(t-\tau)\right\| d \tau \\
= & \frac{1}{\delta t} \int_{0}^{\delta t / 2}\|s(t)-s(t-\tau)\| d \tau+\frac{1}{\delta t} \int_{0}^{\delta t / 2}\|s(t)-s(t-\tau)\| d \tau \\
& +\frac{1}{\delta t} \int_{-\delta t / 2}^{0}\left\|s(t+\tau)-s\left(\left(1-\frac{\delta}{2}\right) t+\tau\right)\right\| d \tau \\
& (26) \quad \frac{1}{\delta t / 2} \int_{0}^{\delta t / 2}\|s(t)-s(t-\tau)\| d \tau+C K_{t}\left(\frac{\delta}{2}\right)^{\alpha} .
\end{aligned}
$$

The same argument leads to

$$
\frac{1}{\delta t} \int_{0}^{\delta t}\|s(t)-s(t+\tau)\| d \tau \leq C K_{(1+\delta) t} \delta^{\alpha} .
$$

The final reasoning is classical: We integrate the inequality

$$
\begin{aligned}
\| s(t) & -s((1-\delta) t) \| \\
& \leq\|s(t)-s(t-\tau)\|+\|s((1-\delta) t)-s((1-\delta) t+(\delta t-\tau))\|
\end{aligned}
$$

over $\tau \in(0, \delta t)$ and obtain

$$
\begin{aligned}
\| s(t)- & s((1-\delta) t) \| \\
& \leq \frac{1}{\delta t} \int_{0}^{\delta t}\|s(t)-s(t-\tau)\| d \tau+\frac{1}{\delta t} \int_{0}^{\delta t}\|s((1-\delta) t)-s((1-\delta) t+\tau)\| d \tau \\
& \quad \leq 27,28) \\
& \leq K_{t} \delta^{\alpha}+C K_{\left(1+(1-\delta)^{-1} \delta\right)(1-\delta) t}\left((1-\delta)^{-1} \delta\right)^{\alpha} \\
& \leq C K_{t} \delta^{\alpha} . \quad \square
\end{aligned}
$$

\section{REFERENCES}

[1] P. Bénilan and M. Crandall, Regularizing effects of homogeneous evolution equations, Amer. J. Math., supplement dedicated to P. Hartmann, 1981, pp. 23-30

[2] S. Campanato, Proprietà di inclusione per spazi di Morrey, Ricerche Mat. 12, 67-86 (1963)

[3] Kuo-Shung Cheng, A regularity theorem for a nonconvex scalar conservation law, J. Differential Equations 61, 79-127 (1986)

[4] J. Conlon, Asymptotic behaviour for a hyperbolic conservation law with periodic initial data, Comm. Pure Appl. Math. 32, 99-112 (1979)

[5] M. Crandall and M. Pierre, Regularizing effects for $u_{t}+A \phi(u)=0$ in $L^{1}$, J. Funct. Anal. 45, 194-212 (1982)

[6] C. Dafermos, Application of the invariance principle for compact processes II. Asymptotic behaviour of solutions of a hyperbolic conservation law, J. Differential Equations 11, 416-424 (1972) 
[7] C. Dafermos, Characteristics in hyperbolic conservation laws, a study of the structure and the asymptotic behaviour of solutions, in Nonlinear Analysis and Mechanics, Vol. 1 (Ed. R. Knops), Pitman, London, 1977

[8] C. Dafermos, Regularity and large time behaviour of solutions of a conservative law without convexity, Proc. Royal Soc. Edin. 99, 201-239 (1985)

[9] J. Glimm and P. Lax, Decay of solutions of systems of nonlinear hyperbolic conservation laws, Mem. Amer. Math. Soc. 101 (1970)

[10] J. Greenberg and D. Tong, Decay of periodic solutions of $\partial u / \partial t+\partial f(u) / \partial x=0$, J. Math. Anal. Appl. 43, 56-71 (1973)

[11] S. N. Kružkov, First order quasi-linear equations in several independent variables, Math. USSR-Sb. 10, 217-243 (1970)

[12] P. Lax, Hyperbolic systems of conservation laws II, Comm. Pure Appl. Math. 10, 537-556 (1957)

[13] P.-L. Lions, B. Perthame, and E. Tadmor, Formulation cinétique des lois de conservation scalaires multi-dimensionelles, C. R. Acad. Sci. Paris Sér. I Math. 312, 97-102 (1991)

[14] T. P. Liu and M. Pierre, Source-solutions and asymptotic behaviour in conservation laws, J. Differential Equations 51, 419-441 (1984)

[15] O. Oleinik, Discontinuous solutions of nonlinear differential equations, Amer. Math. Soc. Transl. Ser. 2 26, 95-172 (1957)

[16] F. Otto, $L^{1}$-contraction and uniqueness for quasilinear elliptic-parabolic equations, J. Differential Equations 131, 20-38 (1996) and C. R. Acad. Sci. Paris Sér. I Math. 321, 1005-1010 (1995)

[17] L. Tartar, Compensated compactness and applications to partial differential equations, In Research Notes in Mathematics, vol. 39, Nonlinear Analysis and Mechanics, Heriot-Watt-Sympos., Vol. 4 (R. J. Knops, ed.) Pitman Press, Boston, London, 1975

[18] K. Zumbrun, Decay rates for nonconvex systems of conservation laws, Comm. Pure Appl. Math. 46, 353-386 (1993) 